\title{
CONCURRENT OPTIMIZATION TECHNIQUE FOR A CONTROLLED STRUCTURE
}

\author{
Brian C. Clendenin and Alexa M. McCulloch ${ }^{*}$ \\ Department of Aerospace Engineering \\ The University of Michigan \\ Ann Arbor, MI 48109
}

\begin{abstract}
A design technique is presented to improve the overall performance of a structural system by simultaneously optimizing the design of the structure and an associated controller. This procedure requires the minimization of a single objective function, subject to dimensional or mass constraints. This is done by including the control and disturbance forces in the equations of motion for the structure and using the eigenfunctions of a uniform structural design as a basis for the eigenfunctions of the optimally designed, nonuniform structure. The results of the concurrent optimization of an Euler-Bernoulli beam and $\mathrm{H}_{2}$ controller are presented. More complicated structures/controllers can be designed using this technique, provided the structure can be modeled as a linear time-invariant plant and the controller synthesis technique involves a single cost functional.
\end{abstract}

\section{INTRODUCTION}

Currently, control-structure interaction is being widely investigated in the aerospace community due to the difficulty of controlling the vibrations of lightweight structures. While structures have traditionally been designed independently of control considerations, significant gains in terms of performance objectives (e.g. vibration suppression or modal damping) may be realized if a structure and associated control system are designed concurrently . The inclusion of the active control system in the optimal design of the structure makes it possible to increase both control authority and disturbance rejection, often decreasing the required mass of the structure. The purpose of this paper is to present a technique for the concurrent optimization of a structure and its controller.

As it is impossible to fully characterize the disturbances acting on a structure, and because of the varying sensitivity of different structures to these unknown disturbances, one would ideally like to design the structure to meet certain performance specifications and then be able to turn on a controller to add to the performance of the structure. The

* Research Fellow, Member AIAA implementation of the controller may affect such parameters as the stiffness, desired damping, shape and mass of the structure. In this work, the ability to concurrently optimally design the structure and its controller to attain the best overall performance of the structure to unknown disturbances is investigated. The performance measure used is the 2-norm of a weighted sum of structural deflections and control effort which will be referred to as the 2-norm of the overall closed loop system.

Multidisciplinary approaches to the optimization of controlled structures have been investigated by various researchers in the past. The problem of controlled flexible space structures was investigated by Woodard et al. ${ }^{1}$ in 1990 . Maghami et al. ${ }^{2}$ formulated the control-structure design problem as a single-objective constrained optimization problem. Starkey et al. ${ }^{3}$ minimized a weighted sum of the structural design parameters and feedback gains and desirable regions for the closed loop eigenvalues. A 10 bar truss example with collocated sensors and actuators was studied by Schmit in $1992^{4}$. Khot used the weight of the structure as an objective function and also investigated the minimization of the Frobenius norm and its relation to the weight of the structure 5 . A multiobjective approach to optimal placement of actuators and sensors in controlaugmented structural optimization was investigated by Sepulveda et al. ${ }^{6}$ in which the structural member sizes and control variables were treated simultaneously as design variables.

\section{STRUCTURAL/CONTROL SYSTEM DESCRIPTION}

A controller is included in the optimal design of the structure by introducing its output to the equation of motion for the structure as a force input. Then, by maximizing the structural response to the controller output and minimizing the structural response to a set of external disturbing forces, optimal structural performance can be achieved.

Copyright 1994 by the American Institute of Aeronautics and Astronautics, Inc. All rights reserved. 
Using a finite element representation of a controlaugmented structure, the dynamic system equations of motion can be written as

$$
[M]]\{\ddot{q}(t)\}+[C]\{\dot{q}(t)\}+[K]\{q(t)\}=\{w(t)\}+\{u(t)\}
$$

where $[M]$ is the system mass matrix, $[C]$ is the system viscous damping matrix, $[\mathrm{K}]$ is the system stiffness matrix, $\{w(t)\}$ is the vector of nodal external excitation forces, $\{u(t)\}$ is the vector of actuator forces and $\{q(t)\}$ is the vector of nodal displacements and rotations.

In order to use the information found by solving the partial differential equations of motion with boundary conditions for the beam (i.e., the natural frequencies and modes of vibration) to design a controller for the structure, a state space model representation of the structural dynamics is preferred. This allows for a standardized controller design approach. We will investigate an example which uses a single inputsingle output (SISO) controller. However, the following derivation is also valid for systems with multiple input-multiple output (MIMO) controllers.

Defining the state space variables as

$$
x(t)=\left\{\begin{array}{c}
q(t) \\
\dot{q}(t)
\end{array}\right\}
$$

The equation of motion can be transformed to state space representation as follows,

$$
\dot{x}(t)=\left[\begin{array}{cc}
{[0]} & {[I]} \\
-[M]^{-1}[K] & -[M]^{-1}[C]
\end{array}\right] x(t)+\left[\begin{array}{c}
{[0]} \\
{[M]^{-1}}
\end{array}\right] u(t)+\left[\begin{array}{c}
{[0]} \\
{[M]^{-1}}
\end{array}\right] w(t)
$$

Thus, the state-space representation of the dynamical system is 7,8

$$
\begin{gathered}
\dot{x}=A x+D_{1} w+B u, \\
y=C x+D_{2} w+D u, \\
\quad \text { and } \\
z=E_{1} x+E_{0} w+E_{2} u .
\end{gathered}
$$

These nine matrices can be written as a partitioned matrix:

$$
G \sim\left[\begin{array}{c|c:c}
A & D_{1} & B \\
\hline E_{1} & E_{0} & E_{2} \\
\hdashline C & D_{2} & D
\end{array}\right]
$$

Here, $x$ is the state vector, a vector of quantities that completely specifies the system. For the structure, $x$ is essentiaily a specification of positions and velocities. The inputs which the controller can freely vary are represented by $u$, while the disturbances over which one has no control are placed in $w$. The output of the system is $y$. A is the dynamics matrix; in this case, it describes how the structure will vibrate. $B$ describes how the inputs act on the system, while $C$ specifies how the outputs are derived from the full state. In some systems, the input directly affects the output; this represented by the matrix $D$. However, for this structure, $D$ will always be zero.

In this formulation, the $D_{1}$ matrix describes how external forces will affect the vibration of the structure, and the $D_{2}$ matrix represents noise in the sensor and actuator measurements. One way of specifying the performance goals is to use $z$, a performance measure, which is a weighted sum of the states and inputs. The maximum amount of effort the actuator can give is specified in the $E_{2}$ matrix, while the relative importance of the states are specified by the $E_{1}$ matrix. In our example, we will assume $E_{0}$, the weighting of the disturbance on the performance, is zero.

\section{PROBLEM STATEMENT}

In this optimization problem, the objective is the minimization of the 2-norm of the complete, closedloop system. $\tilde{G}$ is the transfer function between disturbance $w$ and the observable states of the system, $y$. This function can be formed by combining the plant for the structure, $\mathrm{G}$, and the controller, $\mathrm{G}_{\mathrm{C}}$.

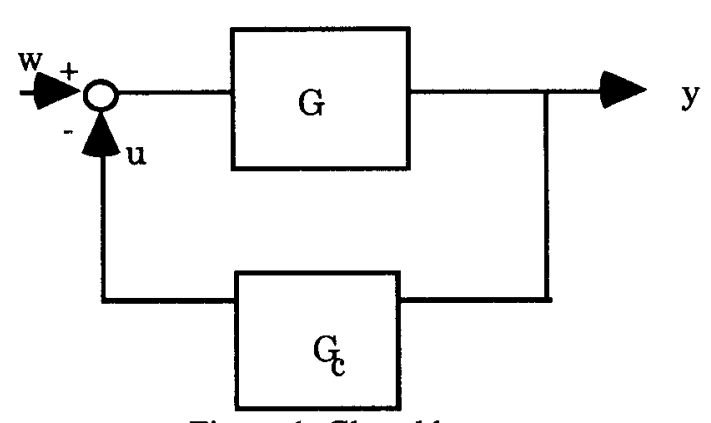

Figure 1: Closed loop system

Finally, the cost functional can be defined as the square of the 2-norm of $\tilde{G}$ :

$$
J=\|\tilde{G}\|_{2}^{2}=\int_{0}^{\infty} z^{T}(t) z(t) d t=\int_{0}^{\infty}\left[\begin{array}{l}
x \\
u
\end{array}\right]^{T} \underbrace{\left[\begin{array}{ll}
E_{1}^{T} E_{1} & E_{1}^{T} E_{2} \\
E_{2}^{T} E_{1} & E_{2}^{T} E_{2}
\end{array}\right]}_{\tilde{R}}\left[\begin{array}{l}
x \\
u
\end{array}\right] d t
$$


The optimization problem becomes

$$
\min _{\nu \in V}\{I\}
$$

subject to

$$
\tilde{A}^{T} P+P \tilde{A}+\tilde{R}=0
$$

(Lyapunov equation for closed loop stability)

and

dimensional and weight constraints on the structure

where

$$
\begin{aligned}
& \text { J: the objective function } \\
& \text { v: vector of design variables } \\
& \text { V: constraint set }
\end{aligned}
$$

$\mathrm{P}$, the observability grammian, is the unique solution to the Lyapunov equation and must be symmetric and positive definite. $\tilde{A}$ is the dynamics matrix of the closed loop system. The design variables for the optimal controller problem are $A_{c}, B_{c}$, and $C_{c}$ which represent the control dynamics. The structural design variables are the dimensions of the structure.

Only recently have sufficient computer resources been available to attempt optimizations over a large parameter space, such as for concurrent optimization of the structure and controller. The optimization is an iterative process which seeks to minimize the controller cost. To take advantage of currently available tools, each iteration of the optimization is done as a four step process. First, a sample structural design is chosen, subject to constraints such as weight, size, or minimum desired stiffness. Then, a model of the structure under consideration is developed. Finally, an optimal controller is synthesized. At this point, the performance measure is calculated for this particular structure/controller pair.

Implementation of this algorithm is accomplished with each step evaluated by a separate computer program. Refer to Figure 2 which illustrates the interaction between steps. The overall control of the process is performed by $\mathrm{EMP}^{9}$, an expert system for generating nonlinear optimization code, which defines the structure to be considered. Once the structure is defined, a basis is defined for the modes of the optimized structure based on known mode shapes for a simplified structure and the coefficients for the new modes as a combination of the basis modes are computed. These mode shapes and frequencies are used by Matlab ${ }^{10}$ to construct an appropriate system model and to find the optimal controller for that model. Matlab then calculates and returns the overall performance of the structure/controller pair to EMP, which uses a Sequential Quadratic Programming (SQP) algorithm to determine the next design step.

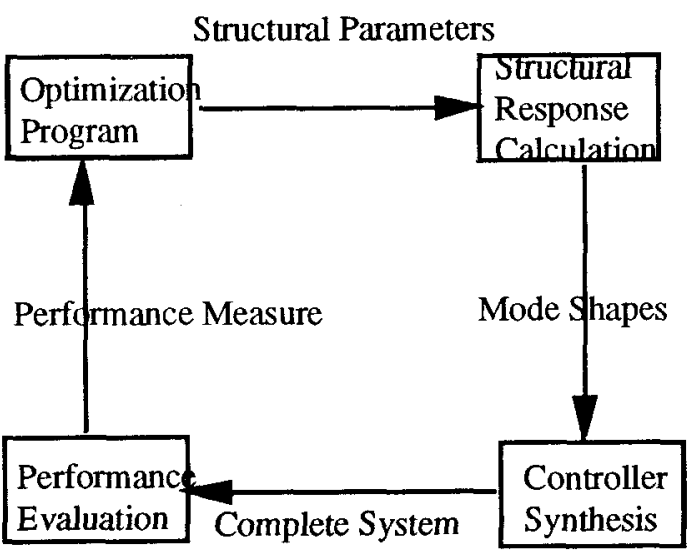

Figure 2: Flow of data in concurrent optimization

\section{EXAMPLE}

We investigate the simultaneous optimization of a Bernoulli-Euler beam and associated $\mathrm{H}_{2}$ controller. This relatively simple example illustrates the interaction between the structure and its control system, as well as performance gains, through a comparison of 1) a uniform beam without controller, 2) a uniform beam with associated optimal controller design, and 3) concurrent optimization of a nonuniform beam and controller. A single input/single output (SISO) controller is designed for the clamped-free beam which has one disturbing force, and one actuator with collocated sensor.

\subsection{Analytical derivation for the uniform beam}

We begin with the Euler-Bernoulli equation for a beam in bending with uniform cross section,

$$
\left(E I w^{\prime \prime}(x, t)\right)^{\prime \prime}+m \ddot{w}(x, t)=f(x, t),
$$

where $w$ is the transverse displacement of the beam, $f$ is a sum of the external forces, $m$ is the linear mass density, $I$ is the moment of inertia, and $E$ is Young's Modulus. It is assumed that the beam is composed of a linearly elastic, isotropic material. Assume that $w$ has the form

$$
w(x, t)=\sum_{i=1}^{\infty} \Omega(t) \Psi_{i}(x)
$$

where the $\Psi_{i}$ satisfy 


$$
E \Gamma \Psi_{i}^{\prime \prime \prime}(x)-\omega_{i}^{2} m \Psi_{i}(x)=0 .
$$

The $\Psi_{i}$ are the mode shapes, and are ordered so that $\omega_{i} \leq \omega_{i+1}$. We substitute the modal decomposition for $w$ into the equation of motion. This yields

$$
\sum_{i=1}^{\infty}\left[E I \Omega_{i}(t) \Psi_{i}^{\prime \prime \prime \prime}(x)+m \ddot{\Omega}_{i}(t) \Psi_{i}(x)\right]=f(x, t) \text {. }
$$

Substituting for $\Psi_{\mathrm{i}}^{\prime \prime \prime \prime}$ and taking the inner product of each side with $\Psi_{j}$ yields

$$
\sum_{i=1}^{\infty}\left[\omega_{i}^{2} \Omega_{i}(t)+\ddot{\Omega}_{i}(t)\right]\left\langle\Psi_{j}(x), m \Psi_{i}(x)\right\rangle=\left\langle\Psi_{j}(x), f(x, t)\right\rangle
$$

Since the $\Psi_{i}$ are orthogonal, $\left\langle\Psi_{j}(x), m \Psi_{i}(x)\right\rangle=0, i \neq j$. So,

$\left[\omega_{j}^{2} \Omega_{j}(t)+\ddot{\Omega}_{j}(t)\right]\left\langle\Psi_{j}(x), m \Psi_{j}(x)\right\rangle=\left\langle\Psi_{j}(x), f(x, t)\right\rangle, \quad \forall j$

At this point, it is convenient to rewrite $f(x, t)$ as a summation of individual terms, which represents a set of independent forces acting on the beam,

Therefore,

$$
F(x, t)=\sum_{k=1}^{m} d_{k}(x) f_{k}(t) .
$$

$$
\omega_{j}{ }^{2} \Omega_{j}(t)+\ddot{\Omega}_{j}(t)=\sum_{k=1}^{m} \frac{\left\langle\Psi_{j}(x) d_{k}(x)\right\rangle}{\left\langle\Psi_{j}(x), m \Psi_{j}(x)\right\rangle} f_{k}(t), \quad \forall j .
$$

For convenience, define

$$
K_{j k}=\frac{\left\langle\Psi_{j}(x), d_{k}(x)\right\rangle}{\left\langle\Psi_{j}(x), m \Psi_{j}(x)\right\rangle} .
$$

Then,

$$
\omega_{j}^{2} \Omega_{j}(t)+\ddot{\Omega}_{j}(t)=\sum_{k=1}^{m} K_{j k} f_{k}(t), \quad \forall j .
$$

The $2^{\text {nd }}$ order inhomogeneous differential equation can be rewritten in matrix form:

$$
\left[\begin{array}{c}
\dot{q}_{1 j}(t) \\
\dot{q}_{2 j}(t)
\end{array}\right]=\left[\begin{array}{cc}
0 & 1 \\
-\omega_{j}^{2} & 0
\end{array}\right]\left[\begin{array}{l}
q_{1 j}(t) \\
q_{2 j}(t)
\end{array}\right]+\left[\begin{array}{ccc}
0 & \cdots & 0 \\
K_{j 1} & \cdots & K_{j m}
\end{array}\right]\left[\begin{array}{c}
f_{1}(t) \\
\vdots \\
f_{m}(t)
\end{array}\right], \forall j
$$

We have represented the jth vibrational mode as a linear system. Now, approximating the entire vibration of the beam by considering $\mathrm{n}$ modes yields

$$
\left[\begin{array}{c}
\dot{\vec{q}}_{1}(t) \\
\dot{\vec{q}}_{2}(t)
\end{array}\right]=\left[\begin{array}{cc}
0 & I \\
-\Omega^{2} & 0
\end{array}\right]\left[\begin{array}{l}
\vec{q}_{1}(t) \\
\vec{q}_{2}(t)
\end{array}\right]+\left[\begin{array}{c}
0 \\
K
\end{array}\right]\left[\begin{array}{c}
f_{1}(t) \\
\vdots \\
f_{m}(t)
\end{array}\right],
$$

where

$$
\begin{gathered}
\Omega=\operatorname{diag}\left(\omega_{1}, \omega_{2}, \cdots, \omega_{n}\right) . \\
K \in \Re^{n \times m}
\end{gathered}
$$

Thus, we have approximated the beam as a finite dimensional linear time invariant system.

\subsection{Controller synthesis}

There are many techniques available to design a controller for a system in the above form. In control theory, it is typical to classify the forces $f_{k}(t)$ as one of two types: inputs from the controller, which we can influence, and disturbing forces, which we cannot influence. Using standard notation and including the effects of damping, the dynamics matrix becomes:

$$
A=\left[\begin{array}{cc}
0 & I \\
-\Omega^{2} & -\mathrm{Z} \Omega
\end{array}\right]
$$

with

$$
\mathrm{Z}=\operatorname{diag}\left(\zeta_{1}, \zeta_{2}, \cdots, \zeta_{n}\right),
$$

where each $\zeta_{i}$ is the damping ratio for each mode. The input $u$ is composed of those $f_{k}(t)$ which represent forces created by the actuators of the controller, while the disturbance $w$ is composed of the other $f_{k}(t)$, over which the controller has no influence. Accordingly, $B$ is made up of those columns associated with the actuator forces, and $D_{l}$, the columns associated with the disturbance forces. We assume the use of velocity sensors positioned at the points of actuation, such that $C=B^{T}$. The performance weighting matrices, $E_{1}$ and $E_{2}$, as well as the matrix representing measurement noise, $D_{2}$, will be discussed in the presentation of the results.

Having constructed a complete model of the beam, we are ready to synthesize a controller for this model. We do an $\mathrm{H}_{2}$, Linear Quadratic Gaussian (LQG), controller synthesis ${ }^{11}$. This technique yields an optimal controller in the sense that, for white noise disturbances, the 2-norm of the performance signal will be minimized. This technique is desirable in that it will yield a controller even in marginal or illconditioned cases, so that, during the iteration process, a controller will almost always be found.

\subsection{Approximate solution for the nonuniform beam}

When considering the nonuniform beam, we again consider the Euler-Bernoulli equation of motion, but allowing $I$ and $m$ to vary with position ${ }^{12}$. As before, we consider a modal decomposition of $w$. 
Unfortunately, it is no longer possible to find closed form equations for the $\Psi_{i}$, which must satisfy

$$
\left(E I(x) \Psi_{i}^{\prime \prime}(x)\right)^{\prime \prime}+\omega_{i}^{2} m(x) \Psi_{i}(x)=0 .
$$

Because this equation cannot be solved in closed form, we need to consider approximations to the actual solution. Approximating the mode shapes $\Psi_{\mathrm{i}}$ by functions $\phi_{i}$, and assuming the $\phi_{i}$ 's to be orthogonal, we can write

$$
\omega_{i}^{2}=\frac{\int_{0}^{L} E I(x)\left(\phi_{i}^{\prime}(x)\right)^{2} d x}{\int_{0}^{L} m(x)\left(\phi_{i}(x)\right)^{2} d x},
$$

which is the familiar Rayleigh quotient 13,14 . The Rayleigh quotient can be calculated for any $\phi_{i}$ satisfying the boundary conditions, and that it is always greater than or equal to the frequency of the solution, with equality holding if and only if the $\phi_{i}$ is the actual solution to the differential equation. Note that the $\phi_{i}$ need to be orthogonal only to find frequencies other than the lowest natural frequency.

The particular approximation that we will consider for each $\Psi_{\mathrm{m}}(\mathrm{x})$, is of the form

$$
\Psi_{m}(x)=\sum_{j=1}^{\infty} c_{m j} \phi_{j}(x),
$$

where these $\phi_{j}$ are the mode shapes for the uniform beam.

Substituting this form for $\Psi_{m}(x)$ into the Rayleigh quotient, we have, for the numerator

$$
E \sum_{j=1}^{\infty} \sum_{k=1}^{\infty} c_{m j} c_{m k} \int_{0}^{L} I(x) \phi_{j}^{\prime \prime}(x) \phi_{k}^{\prime \prime}(x) d x .
$$

This expression is still extremely difficult to integrate, so we will consider a design that varies piecewise over the length of the beam. If the width on interval $i$ is $b_{j}$ and the height is $h_{j}$, this integral reduces to

$$
E \sum_{i} \sum_{j=1}^{\infty} \sum_{k=1}^{\infty} c_{m j} c_{m k} b_{i} h_{i} \int_{x_{i}}^{x_{i+1}} \phi_{j}^{\prime \prime}(x) \phi_{k}^{\prime \prime}(x) d x .
$$

Similarly, the denominator can be written as

$$
E \sum_{i} \sum_{j=1}^{\infty} \sum_{k=1}^{\infty} c_{m j} c_{m k} b_{i} h_{i} \int_{x_{i}}^{x_{i+1}} \phi_{j}(x) \phi_{k}(x) d x .
$$

The expression for orthogonality of the $\Psi_{i}$ is derived in the same manner, which yields
$E \sum_{i} \sum_{j=1}^{\infty} \sum_{k=1}^{\infty} c_{p j} c_{q k} b_{i} h_{i} \int_{x_{i}}^{x_{i+1}} \phi_{j}(x) \phi_{k}(x) d x=0, \quad p \neq q$

This orthogonality condition must be satisfied in order to find the higher modes of the nonuniform beam.

We can now build a complete model of the beam as was done for the uniform beam, using the Rayleigh quotient to find the natural frequencies and the approximate mode shapes. As for the uniform beam, we will be using $\mathrm{H}_{2}$ (LQG) controller synthesis. The only difference is the plant model used for controller design. The plant model for the nonuniform beam has more degrees of freedom than the uniform beam model, allowing the investigation of a larger class of systems.

\subsection{Alternate methods}

We consider a problem that minimizes the 2-norm of the modal deflections; minimizing deflections at the point of disturbance, while maximizing deflections due to the control force. This results in a much simpler model than others considered, but it may also yield less accurate results; displacements, while related to the energy, are only a portion of the modal energy of the structure. However, this is a very simple approach that seems to be an appropriate first step towards developing a more exact method.

As an alternative, we could choose the modal energy approach, as it models the structure reasonably well, and it also gives ready insight into how a structure might be designed to have a better response to control inputs and disturbances. This method which uses both position and velocity. In the future, this would be a likely candidate for additional research.

Another alternative modeling approach is Statistical Energy Analysis (SEA), which models the flow of energy between the subsystems of a structure 15,16 . This approach would be to be especially effective in a large, complicated structure, such as a truss. However, SEA is a more complex theory, and there is very little insight into how the dynamical and geometrical parameters affect the flow of energy. This lack of insight makes it difficult to analyze the resulting system and also creates potential problems with the robustness of the design process.

Although we have considered a $\mathrm{H}_{2}$ (LQG) controller for this example, other controllers may have worked as well. Such controllers could include an $\mathrm{H}_{\infty}$ controller that minimizes the o-norm of the disturbance-to-performance transfer function or a 
LQR controller that maintains a static gain over all frequencies.

We solve the problem for each of three cases: uniform beam, uniform beam with controller, and nonuniform beam with controller.

\subsection{Sample uniform beam}

We examine a uniform beam with width and height equal to $10 \mathrm{~cm}$. As shown in Figure 2, the disturbance force, $w$, is acting at the mid-span of the beam, while the actuator is acting at the end.

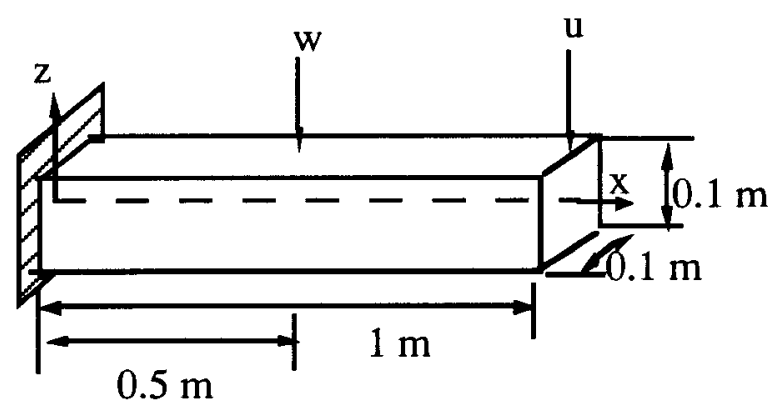

Figure 3: Sample uniform beam

A problem that one encounters in every control design problem is just how much information to include in the model of the system one is trying to control; in this case, how big the dynamics matrix and state vector should be. The analytic model we have been dealing with gives no clear indication regarding how many modes to include, but we will take the first three modes ${ }^{17}$, disregarding the rest for computational purposes. We assume that the higher modes will contribute less to the vibrational response of the beam because of the frequency content of the disturbance. However, one must be careful when designing a controller for such a simplified system; an unmodeled mode can be forced unstable by controller excitation. Each mode is assumed to have $1 \%$ damping (damping ratio of 0.01 ).

Having chosen the size of our beam and the number of modes to account for, we can find the natural frequencies and the coefficients for the $B$ and $D_{1}$ matrices. These values are presented in Table 1 .

Table 1: Natural frequencies and vibrational responses for the first three modes

\begin{tabular}{|c|c|c|c|}
\hline $\begin{array}{c}\text { Mode } \\
\text { Number }\end{array}$ & $\begin{array}{c}\text { Natrual } \\
\text { Frequency }\end{array}$ & $\begin{array}{c}\text { Input } \\
\text { Response }\end{array}$ & $\begin{array}{c}\text { Disturb. } \\
\text { Response }\end{array}$ \\
\hline 1 & $40 \mathrm{~Hz}$ & -0.448 & -0.152 \\
2 & $250 \mathrm{~Hz}$ & 0.448 & -0.319 \\
3 & $700 \mathrm{~Hz}$ & -0.448 & -0.009 \\
\hline
\end{tabular}

4.6 Sample uniform beam with optimal controller

As a natural performance measurement, we have chosen to minimize the 2-norm of the time response of the deflection of the beam to a white noise disturbance force placed at the midpoint of the beam, or, equivalently, to minimize the 2-norm of the transfer function from the disturbance to the performance measurement (displacement). The performance measurement is chosen through the use of the $E_{1}$ and $E_{2}$ matrices. For this problem,

$$
\begin{gathered}
E_{1}=\left[\begin{array}{cccccc}
3000 & 9000 & 3000 & 0 & 0 & 0 \\
0 & 0 & 0 & 0 & 0 & 0
\end{array}\right] \\
E_{2}=\left[\begin{array}{l}
0 \\
1
\end{array}\right]
\end{gathered}
$$

This places a premium on the vibrational amplitude of the modes, and allows the controller to exert a relatively large amount of effort. In other words, we are claiming that we have fairly powerful actuators and want the vibration to be reduced dramatically. The zeros in the matrices are due to the fact that the two performance variables, weighting the state and control effort, should be independent of each other. We set our measurement noise to be 0.0004 , orders of magnitude smaller than the disturbance constants. In other words, compared to the disturbing forces, our sensors are quite accurate.

With the complete system model in hand, we solve for the optimal controller using Matlab. As described above, a closed form solution for the optimal $\mathrm{H}_{2}$ controller exists.

\subsection{Concurrently optimized nonuniform beam and controller}

We generalize the techniques to allow for a beam of varying but solid cross section. We examine the design of a piecewise uniform beam, considering a beam divided into ten segments of length $0.1 \mathrm{~m}$, with width $b_{i}$ and height $h_{i}, i=1,2, \ldots 10$. This entailes the use of the approximations derived above. In addition, we also use polynomials to approximate the mode shapes of the uniform beam, to facilitate the necessary integration. We used a Taylor series expansion of up to twenty terms to approximate the mode shapes, allowing the integration to be carried out easily.

The frequencies of the nonuniform beam vary much more widely than the possible frequencies of the uniform beam. This required the $E_{I}$ matrix to be chosen differently. In the example chosen, we selected 

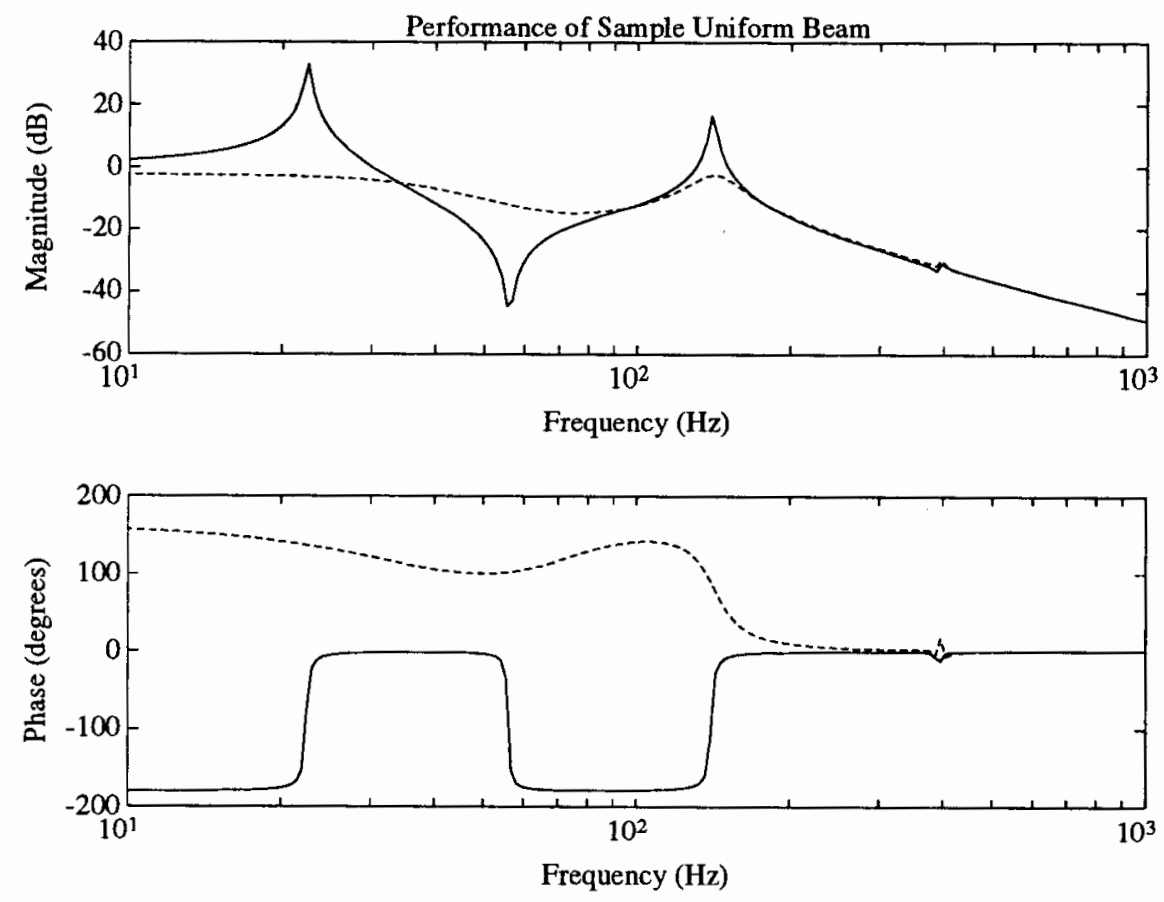

Figure 4: Performance of the sample uniform beam and optimal $\mathrm{H}_{2}$ controller
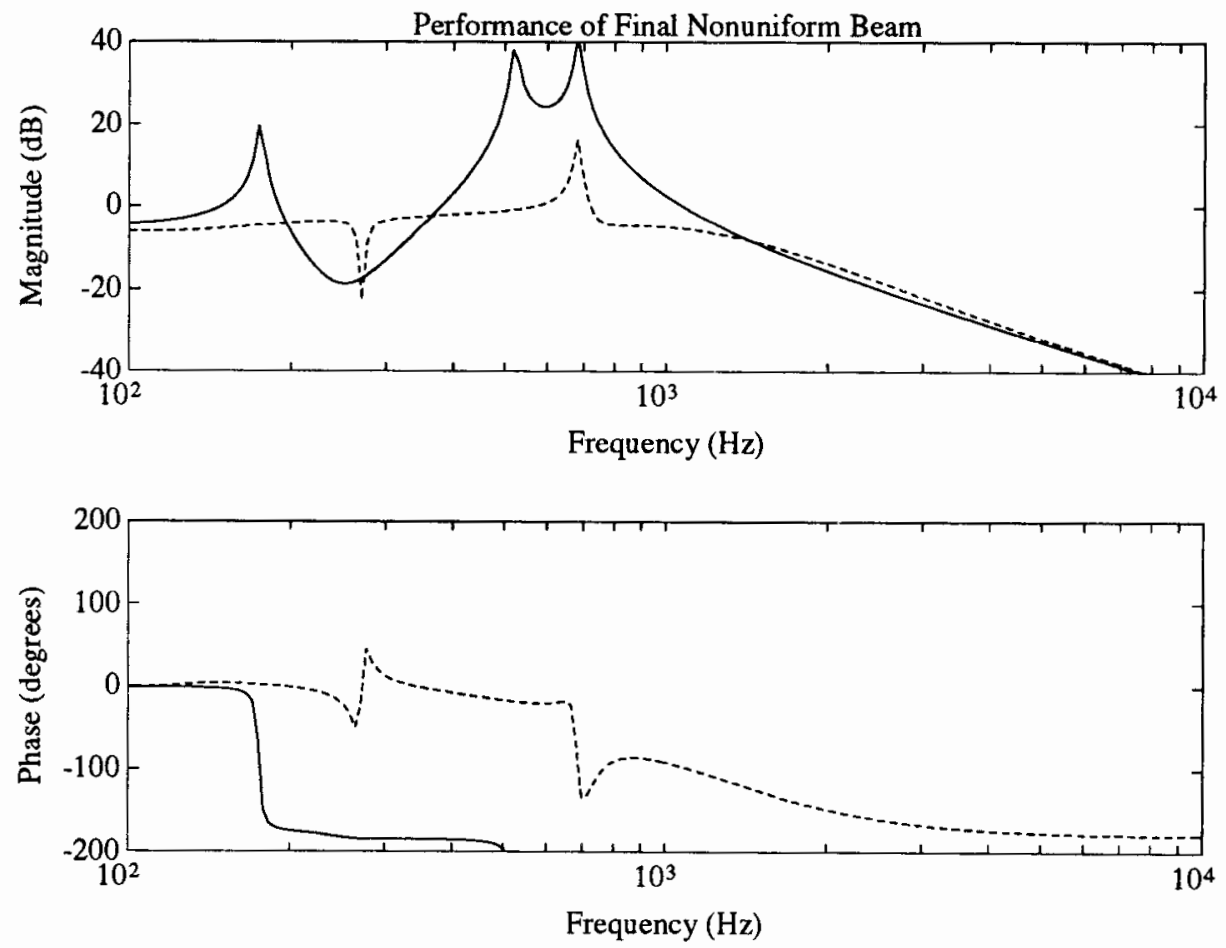

Figure 5: Performance of the optimal nonuniform beam and $\mathrm{H}_{2}$ controller 


$$
E_{1}=\left[\begin{array}{cccccc}
\frac{\omega_{1}{ }^{2}}{10} & \frac{\omega_{2}^{2}}{10} & \frac{\omega_{3}^{2}}{10} & 0 & 0 & 0 \\
0 & 0 & 0 & 0 & 0 & 0
\end{array}\right]
$$

The other values remained identical to the uniform beam. The constraints for this optimization problem were that the approximations to the nonuniform mode shapes had to be orthogonal to each other, as well as normalized. We also had a resource constraint of $0.007 \mathrm{~m}^{3}$, and in each interval, the width and height were forced to lie between $1 \mathrm{~cm}$ and $10 \mathrm{~cm}$.

The problem statement becomes:

$$
\min _{\bar{b}, \bar{h}, \bar{c}}\left(\|G\|_{2}\right)
$$

subject to

$$
\begin{aligned}
& \text { 1) closed loop stability } \\
& \text { 2) volume }-0.007 \mathrm{~m}^{3} \leq 0 \\
& \text { 3) orthogonality of the mode shapes }
\end{aligned}
$$

\section{RESULTS}

In both optimization problems defined above (uniform beam and nonuniform beam), concurrent optimization of the structure-controller pair yields significantly improved performance over the baseline design. Not only did the $\mathrm{H}_{2}$ response improve, but the $\mathrm{H}_{\infty}$ response (peak response) is significantly improved as well. In Figure 4, we show the effect of adding a controller to a benchmark beam. Figure 5 shows the performance of the optimal nonuniform beam/controller system. The performance of the nonuniform beam is similar to the uniform beam case, but the weight of the structure is decreased considerably. Figure 6 shows the nonuniform beam. The resulting design is similar to that expected. The beam is thicker near the root and near the disturbance input, and thinner everywhere else.

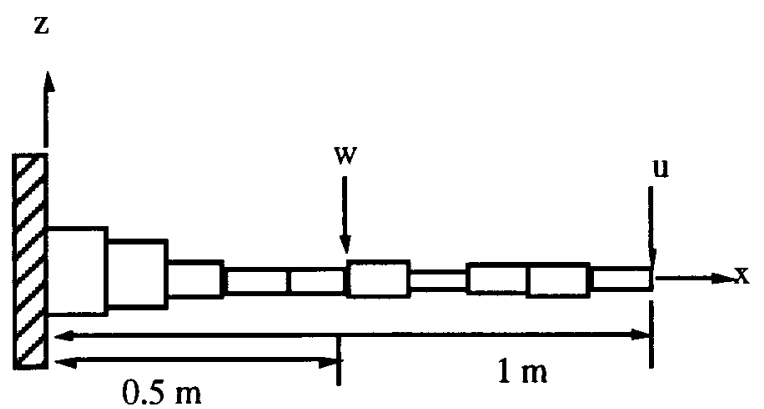

Figure 6: Optimal nonuniform beam
Table 2: Shape of each element for final nonuniform

beam

\begin{tabular}{|l|l|l|}
\hline Interval & Width (m) & Height (m) \\
\hline 1 & 0.020 & 0.063 \\
2 & 0.010 & 0.043 \\
3 & 0.016 & 0.017 \\
4 & 0.011 & 0.013 \\
5 & 0.061 & 0.013 \\
6 & 0.042 & 0.016 \\
7 & 0.014 & 0.011 \\
8 & 0.023 & 0.015 \\
9 & 0.015 & 0.014 \\
10 & 0.014 & 0.011 \\
\hline
\end{tabular}

\section{CONCLUSIONS}

The method presented here may have a number of applications. It will be most useful in the synthesis of new structures, designed with integrated active control systems. Concurrent optimization will yield extremely efficient structures that meet stringent stiffness requirements. This technique may also be used to guide the redesign or modification of existing structures to be retrofitted with active controllers.

There are a number of issues yet to be addressed. Primary among them is a need to modify the formulation to prevent mode shifting and guarantee sufficient spacing between modes. Other extensions involve refinement of the structural model to allow more accurate mode shape determination, inclusion of more structural modes in the dynamic model, and generalization of the structural model to allow more complex cross-sectional variations along the length of the beam.

More complex structures and other optimal controllers can be designed using this technique, provided that the overall performance can be expressed in terms of a scalar cost functional. One must be able to model the structure in a form compatible with the controller synthesis technique, and the resulting cost functional must have certain mathematical properties (continuity is sufficient, convexity is desirable).

Extensions to this study will include investigating distributed actuators and sensors to accommodate smart structures that use piezoelectric (PZT) actuators and polyvinylidene fluoride (PVDF) sensors, which are currently being investigated and used for aerospace applications. 


\section{ACKNOWLEDGEMENTS}

We would like to thank Dr. John E. Taylor, Dr. Dennis S. Bernstein and Dr. Panos Papalambros for their support and guidance.

\section{REFERENCES}

1. Woodard, S. E. and S. L. Padula, "A Multidisciplinary Approach to Optimization of Controlled Space Structures," Third Air Forcel NASA Symposium on Recent Advances in Multidisciplinary Analysis and Optimization, September 24-26, 1990.

2. Maghami, P. G., S. M. Joshi, J. E. Walz, and E. S. Armstrong, "Integrated Controls-Structures Design Methodology Development for a Class of Flexible Spacecraft," Third Air Forcel NASA Symposium on Recent Advances in Multidisciplinary Analysis and Optimization, September 24-26, 1990.

3. Starkey, J. M. and P. M. Kelecy, "Simultaneous Structural and Control Design Using Constraint Functions," ASME Journal of Mechanisms, Transmissions, and Automation in Design, Vol. 110, p65-72, March 1988.

4. Schmit, L. A., " Control Design Variable Linking for Optimization of Structural/Control Systems," AIAA Journal, Vol. 30, num. 7, p1892-900, July 1992.

5. Khot, N. S., " Optimum Structure and Control Design," Computational Mechanics '88 Theory and Applications Proceedings of the International Conference on Computational Engineering Science, Springer-Verlag, 1-4 vol. 2, p434, 1988.

6. Sepulveda, A. E., and L. A. Schmit, Jr., "Optimal Placement of Actuators and Sensors in ControlAugmented Structural Optimization," International Journal Numerical Methods in Engineering, Vol. 32, p1165-1187, 1991.
7. Chen, C. T., Introduction to Linear System Theory, Holt, Rhinehart, Winston, 1984.

8. Doyle, J. C., Francis, B. A., and Tannenbaum, A. R., Feedback Control Theory, Macmillan, 1992.

9. Schittkowski, K. EMP: An Expert System for Mathematical Programming, Technical Report, Mathematics Institute, Universitaet Bayreuth, 1985.

10. The Student Edition of MATLAB for Macintosh Computers. The MathWorks, Inc., Prentice-Hall, Englewood Cliffs, 1992.

11. Maciejowski, J. M., Multivariable Feedback Design Addison-Wesley, 1989.

12. Meirovitch, L., Analytic Methods in Vibrations. MacMillan, 1967.

13. Bendsoe, M.P., N. Olhoff, and J. E. Taylor, "A Variational Formulation for Multicriteria Structural Optimization," Journal of Structural Mechanics, 11(4), pp. 523-544, 1983-1984.

14. Taylor, J. E., "Distributed Parameter Optimal Structural Design: Some Basic Problem Formulations and Their Application," in M. P. Bendsoe, and C. A. Mota Soares, eds., Topology Design of Structures. NATO ASI, Sesimbra, Portugal, 1992.

15. Kishimoto, Y., and D. S. Bernstein "Thermodynamic Modeling of Interconnected Systems: I. Conservative Coupling," Department of Aerospace Engineering, University of Michigan, February 1993.

16. Miller, D. W., S. R. Hall, and A. H. von Flotow, "Optimal Control of Power Flow at Structural Junctions," Journal of Sound and Vibration, vol. 140 , pp. 475-497, 1990.

17. Blevins, R. D., Formulas for Natural Frequency and Mode Shapes, Van Nostrand, 1979. 